\title{
Presente y futuro latinoamericano de la enseñanza y el aprendizaje en entornos virtuales referidos a educación universitaria
}

\section{Latin American Present and Future of Teaching and Learning in Virtual Environments in Relation to Higher Education}

\author{
Beatriz Fainholc \\ Universidad Nacional de La Plata-CEDIPROE. Argentina. \\ bfainhol@gmail.com
}

\begin{abstract}
Resumen
El sistema educación virtual, en especial de nivel superior direccionado para la producción de conocimiento en América Latina, si bien ha demostrado avances, aún presenta graves debilidades con una baja incorporación de Internet, TIC y derivados. No superó la baja calidad educativa general y a distancia, no interviniendo positivamente con sus impactos en la vulnerabilidad y riesgos sociales existentes.
\end{abstract}

Debido a los resultados obtenidos, se necesita una revisita conceptual y metodológica de las instituciones, programas de educación superior a distancia virtual, para una consecuente redefinición de concepciones que sostienen el diseño, implementación y evaluación de propuestas en el área, de una fuerte y articulada proyección socioeconómica, cultural y formativa para estos países, las que no ocultan las luchas por el poder que decide acerca de lo nombrado.

Una perspectiva educativa genuina de la virtualidad, podría ser una protagonista central en la sociedad informacional de redes para superar la racionalidad técnico-instrumental de los marcos epistémico-político-académicos y sociales de resultados socioeducativos inequitativos.

La tecnología educacional es una variable dependiente / interviniente en la formación en general de las personas y del sector científico tecnológico de un país, región, etc., que debería formar una ciudadanía consciente y con competencias de agencia, que poco lo ejecuta.

\section{Palabras claves}

Sociedad de la información. Educación superior virtual. Poder, Producción y uso de conocimiento digital, Reformulación de concepciones pedagógicas científico- tecnológicas. Situación de América Latina.

\begin{abstract}
The virtual educational system, in particular referred to Higher Education towards knowledge production in Latin America is in progress. However, it still shows strong weaknesses with poor Internet connection, ICT and derivate devices and formats. Moreover, it has not been able to overcome the low educational quality it offers in general and in distance programs; or to study if the impact of training courses has improved the high social vulnerability, the inequity results and their known risks.
\end{abstract}

Based on these appreciations, it is necessary, among many topics, to revisit the conceptual and methodological framework of electronic educational proposals, and the organizational and institutional conceptions of universities, including distance/virtual Higher Education programs, in order to redefine their design, implementation and evaluation because of the strong socio-economic, cultural and formative projections that these countries need for 
their development when adopting those educational proposals. The intervening variable of the power structure which many times, decides about those issues is also considered.

A genuine educational perspective of virtuality should have a crucial role in the information and network society, through overcoming the technical-instrumental rationality with political-economical, academic and socio-epistemic framework change.

The educational technology is a depending/intervening variable to the formation of people, as it is known. Besides, the scientific and technology sectors are highly relevant for a country, region, etc., related to the development of an aware citizenry and their agency competences, which they usually lack.

\section{Key words}

Information Society. Higher virtual education. Production, Use of digital knowledge. Reformulation of pedagogic-scientific-technological conceptions. Latin American countries.

\section{Introducción}

La presente evolución-revolución tecnológico- electrónica disruptiva que se vive, hace necesario detenerse para su reflexión. Diríamos una reinvención, reestructuración meticulosa de todas las instituciones, organizaciones y programas educativos. Las preguntas inquietantes son: en interés de quiénes va la tecnología, de todos/as, con qué sentido y fines, y otras muchas más, que, en realidad se han planteado desde siempre: se remontan a la Ilustración, donde se creía que la reestructuración progresiva de la educación robustecería la democracia, que se reviven hoy.

En América Latina, como en otras regiones del mundo, las tecnologías de la información y la comunicación, no sólo han puesto en evidencia las fallas del sistema educativo y universitario, sino que al mismo tiempo se muestran que son parte de la alternativa de solución para una propuesta de un modelo inscripto en la cultura digital con sus características positivas (por lo menos en teoría, las hay negativas) de horizontalidad, transparencia, colaboración, construcción colectiva, y otras.

Es decir, hoy, las presiones para el cambio proceden, entre diversos rubros, de la tecnología virtual y de la economía global, y los revisionismos de los acostumbrados principios, teorías y conceptos socio-políticos, culturales y educativos reformistas, que a pesar de rememorar sus esfuerzos, no alcanzan como propuesta de parámetros de comprensión de lo que ocurre en la sociedad informacional y la cultura digital.

Los cambios complejos y vertiginosos hacen previsible que sean necesarias otras formas de organización académica y administrativas para la educación en general y de nivel superior, para apoyar los nuevos perfiles requeridos por los nuevos e inciertos tiempos. Este diseño debería apostar a muchas tentativas de solución, y no una sola, acordes a las demandas de la cultura digital.

\section{¿Dónde estamos?}

El siglo XXI está indicando un quiebre de perspectivas de la anterior era moderna, de sus concepciones y valores. La que la reemplaza es la conocida como posmoderna, hoy post-post e hiper-moderna, de grandes crisis, económico-políticas del capitalismo tardío,

Presente y futuro latinoamericano de la enseñanza y el aprendizaje en entornos virtuales referidos a educación universitaria. Beatriz Fainholc. 
con situaciones extremistas violentas, donde la comunicación inmediata, reticular y fluida, -aunque precaria- une, unifica, y al mismo tiempo refleja la exclusión ${ }^{1}$ y fragmentación social en el mundo, entre otros rasgos.

La aceleración histórica y el avance arrollador en este segundo decenio del siglo XXI, de desorden global, de los nuevos activismos políticos, de la explosión virtual de datos (big data), la intercomunicación de cosas y movimientos (Internet de las cosas), dada por sensores, y otros, son los grandes y preocupantes temas contemporáneos, que muestran anacronismos del sector educativo, el cual debería haber sido el más consciente y anticipador, estando en sus manos la formación de personas.

Así, en referencia a la incorporación de las TIC y derivados a la educación en general en América Latina, ésta ha sido y es despareja, heterogénea, y de tardanza, aunque últimamente los países de la región, se hayan esforzado ${ }^{2}$ en ello tanto en su acceso como en la alfabetización tecnológica. Sin embargo, el desarrollo de programas de educación a distancia, incluido el de algunas universidades en esta modalidad, mantienen perfiles poco redefinidos aún siendo abiertos (Moodle, MOOC, etc.).

Los ambientes virtuales de aprendizaje continúan los modelos de los EVA conocidos, separados por un contraste de estos programas (muchos de ellos muy loables) con poca consideración de la situación de exclusión ${ }^{3}$, fragmentación, asimetrías ${ }^{4}$ sociales y pobreza de dichos países, que evapora los esfuerzos educativos para formar gente en desenvolverse en la sociedad de la información, con pertinencia, de manera pro-activa y responsable.

\section{¿Cuál es el problema?}

En base a la situación referida de los países de América Latina (y de la Argentina, desde donde escribe la autora), la misión de la ciencia, la tecnología y la educación no atiende debidamente la situación referida. Aviso: toda comparación entre países no cabe en este planteo de rescatar la singularidad de las políticas implementadas de cada país en América Latina.

Sin embargo, el mayor desfasaje se relaciona con el rol otorgado y practicado en la educación, más allá de los productos y servicios distribuidos que circulan posibilitados por las interfaces y redes. Más allá de valorar que sean altamente participativos, la interacción social frente al nutrido conocimiento existente, en general, encubre modos míticos, naturalizados e invisibles, que no apelan a la reflexividad del pensamiento -

\footnotetext{
${ }^{1}$ El paradigma de la desigualdad no se define hoy tanto por estar en los márgenes de la producción, sino por estar excluidos de los flujos de la circulación global de la información

${ }^{2}$ La Cumbre Mundial sobre la Sociedad de la Información ha trazado 10 objetivos educativos a ser alcanzados por América Latina, en 2015, en donde dos se refieren a educación y tecnologías de la sociedad de la información.

${ }^{3}$ La "inclusión o inserción social", apunta a que todos/as alcancen todas las dimensiones de una vida digna. Ello hoy se halla atravesado por la posibilidad educativa de producción y uso de herramientas telemáticas participativas, la lectura crítica de pantallas y, el ejercicio de competencias digitales.

4 Piketty, T. (2014). El capital en el siglo XXI. México: FCE.
}

Presente y futuro latinoamericano de la enseñanza y el aprendizaje en entornos virtuales referidos a educación universitaria. Beatriz Fainholc. 
hoy digital rizomático y no lineal- que se cree, redundaría en un entendimiento amplio y competente que innove en propuestas pertinentes de todo tipo.

\section{Argumentación}

\section{América Latina es una dimensión de lo aún no consciente y lo aún no devenido}

Estamos frente a enormes desafíos y oportunidades para la educación superior en general y en especial, virtual, para América Latina, si se entiende que esta modalidad podría ayudar a sus sociedades y economías, a crear valor y formar personas resolutivas y creativas preparadas según las demandas actuales. La tecnología hoy lo permite siempre y cuando se reconozcan las limitaciones teóricas y prácticas de Internet, las TIC y sus derivados, sin soslayar sus fortalezas: mucha literatura especializada así lo enuncia.

Habría que rescatar algunos componentes (que son desafíos ${ }^{5}$ muy significativos) referidos a la educación virtual) como es el del enfoque ${ }^{6}$ de la enseñanza, el aprendizaje e investigación iluminadora de propuestas alternativas, para la educación superior general y virtual en América Latina para los próximos años.

\section{Una breve radiografía de la educación virtual de América Latina}

Lamentablemente, la incapacidad de regular las distopías de la realidad actual, con crisis de la economía en su conjunto, y una desaceleración de la tasa de innovación, como input y resultado de las diversas acciones socio-políticas: por ejemplo, investigación científico-tecnológica glocales, aplicable a la producción socialmente útil con la intervención equitativa de la variable educativa, formará genuinamente y no cooptada, personas calificadas.

El sentido de la educación y en consecuencia del significado de decisiones científicotecnológicas es demostrado en los análisis insuficientes que no aplican un enfoque relacional, al suponer que sólo una dimensión y un instrumento, resolvería la problemática de la exclusión social. Aún quedan sin considerar las dimensiones estructurales socio-culturales, económico-políticas y éticas a estudiar y considerar. Por ejemplo, las limitaciones de la democracia sostenidas por las vigentes políticas públicas equitativas, que cierra las posibilidades de justicia.

\footnotetext{
5 Perspectivas Tecnológicas Educación Superior en América Latina 2013-2018 Un Análisis Regional del Informe Horizon NMC (2013) Publicado por The New Media Consortium (NMC). Centro Superior para la Enseñanza Virtual (CSEV), y Virtual Educa.

Consultado 15- nov-2015 http://www.nmc.org/pdf/2013-technology-outlook-latin-america-ES.pdf

${ }^{6}$ El marco inter/transdisciplinario, de políticas de ciencia y tecnología y de educación, de los contextos de investigación articulados con la enseñanza de los centros de educación superior, y de Investigación y Desarrollo, constituyen los ejes fundamentales de la organización, generación de conocimiento apropiado, tal las necesidades de conocimiento socialmente pertinente y útil.
}

Presente y futuro latinoamericano de la enseñanza y el aprendizaje en entornos virtuales referidos a educación universitaria. Beatriz Fainholc. 
Superar fragmentaciones y esquematismos son diversos prerrequisitos para un desarrollo que configure puentes para superar la desigualdad.

Algunas argumentaciones aparecen a continuación, que sirven de respaldo para seguir pensando.

1) Crear modelos flexibles de educación superior híbridos que incluyan aprendizajes off-online, presencial y mediados por TIC y derivados, donde los decisores de las políticas generales e institucionales, evalúen profundamente los programas en la acción, con una inversión sostenida de planes estratégicos, que compartan honestamente valiosos recursos, etc., integrados a las existentes formas ya integradas o a ser integradas en nuevos programas educativos formales, no formales e informales, de modo articulado.

2) Como la mayoría de los académicos poco están utilizando nuevas tecnologías electrónicas significativas para el aprendizaje y la enseñanza, ni para organizar sus investigaciones presenciales y a distancia, se debería concientizar acerca de ello con tiempos y espacios dedicados. Muchos investigadores no han sido formados en las técnicas básicas de enseñanza digitales, y deberían ser entrenados en la práctica como parte del desarrollo profesional, académico, etc.: ello debería ser aprendido y enseñado. Se alegan factores como falta de tiempo, cambio actitudinal y de bajas expectativas frente a las TIC, debido a los resultados publicados de poco peso de aquéllas en el mejoramiento del aprendizaje, - lo cual es cierto-, y otros.

3) Son infinitos los estudios (que sería infinito nombrar aquí) que refieren a fortalecer la formación pedagógica crítica continua general y al uso reflexivo de las TIC para estimular la innovación colectiva creativa a través de metodologías socio-didáctico y culturales apropiadas $^{7}$ al nivel representacional de los estudiantes y del contexto/coyunturas sociales. En términos llanos, esta formación no está suficientemente integrada en los planes de estudio en general -en muchísimos países del mundo, así y en América Latina-, de las disciplinas académicas superiores universitarias. Esto puede deberse a aferrarse a lo conocido -aunque anacrónico-, a la carencia generalizada de desarrollo profesional para formadores en esta área, cargos de bajos aranceles y no dedicación full-time, falta de decisiones claras, y otros.

4) Las instituciones universitarias en América Latina, no han tenido en cuenta el cambio, aumento de especialización de los nuevos perfiles laborales con las competencias requeridas por los ambientes digitales ${ }^{8}$, que podrían ser aplicables y apropiables para casi todas las tareas generales, sociales y educativas. Aunque todavía estemos lejos de que la enseñanza de competencias digitales verificables se vincule con una manera nueva y reflexiva de pensar los estándares de las herramientas y plataformas, -a pesar de ser en cierto modo efímeros-, éstas necesitan ser probadas experiencialmente. Claro que, apelando a los principios de cautela, precaución y prudencia en las evaluaciones tecnológicas y aplicaciones en la toma de decisiones

\footnotetext{
${ }^{7}$ Fainholc, B. (2013). Una tecnología educativa apropiada y crítica. Buenos Aires: Humanitas

8 Ilustrativamente ver: Diez Retos Significativos para la Adopción de Tecnología. 2013, NMC Un Análisis

Regional del Informe Horizon del NMC. Se pueden consultar los boletines actuales.
}

Presente y futuro latinoamericano de la enseñanza y el aprendizaje en entornos virtuales referidos a educación universitaria. Beatriz Fainholc. 
educativsa, para la inversión sostenida en ellasy otros rubros, y no dejar al libre albedrío transferencias tecnológicas, usos indiscriminados $\mathrm{y}$ hasta peligroso, abusos consumísticos vacíos de sentido, etc.

5) Un argumento tal vez descuidado - aunque se dice lo contrario, sobre todo para zonas rurales, está referido a la infraestructura existente, sin la cual es imposible soportar la conectividad, cualquier software, etc. Si bien los teléfonos móviles son aliados para su uso educativo y producción de propuestas locales, las instituciones de educación superior latinoamericanas todavía carecen de la infraestructura necesaria,- la que se está abaratando-, y que podría actuar para promover el aprendizaje ubicuo ${ }^{9}$ de estos tiempos.

6) La renovación/redefinición continua de los paradigmas universitarios vigentes trasvasados para una innovación en la enseñanza virtual, provoca nuevas percepciones, lo que fortifica el espíritu de transformación. Los contenidos científicos súper actualizados articulados con metodologías y actividades de aprendizaje inspiran superar lo prescrito tradicional, es reconocer el transversal de la tecnología en todas las esferas.

7) La importancia de la investigación general, y en la y para la acción, está ampliamente reconocida. Las estructuras organizativas actuales poco la promueven porque nada se valora y recompensa. Lo mismo ocurre con el lugar de la experimentación o la aplicación innovadora de tecnologías, la cual se considera frecuentemente fuera de las funciones del investigador o científico y, por tanto, se desalientan cuando es sabido que la investigación científica cabalga junto con la investigación tecnológica al expandir la "humanidades digitales", es decir, la integración de las ciencias humanas con las informáticas en todo sector de estudio, y por ende, en el ámbito de la enseñanza y el aprendizaje virtual, si hubiera ofertas superiores de esta modalidad.

8) Otro argumento aceptado y recomendado en toda reunión internacional, para transformar el estado de la educación superior en Latinoamérica, con conceptos y métodos según pedagogías emergentes on-line ${ }^{10}$, es entender sus problemas con un abordaje interdisciplinario y un enfoque sistémico-recursivo-holista y relacional de entendimiento del mundo, la vida y la persona. A partir de este marco, diseñar, rediseñar e implementar los programas de aprendizaje electrónico puro (e-learning) y mixto $^{11}$, presencial y mediado por Internet, TIC y derivados (blended learning), tan en auge. Muchos programas así ya lo demuestran, al articular nuevas concepciones pedagógicas y tecnológicas, contextuadas socio-históricamente, en la convicción que coadyuva a la mejora de la situación de la educación virtual en América Latina (aunque no sólo de esta región).

\footnotetext{
${ }^{9}$ Burbules, N. (2012) aprendizaje ubicuo y el futuro de la enseñanza Revista Encounters/Encuentros/Rencontres on Education Vol. 13, 2012, 3 - 14

${ }^{10}$ Fainholc. B. (2015) Epistemologías emergentes. http://webquestorgar.blogspot.com.ar/2015/11/epistemologias-emergentes-y-educacion.html ${ }^{11}$ Fainholc, B. (2012). Aprendizaje electrónico mixto. El blended learning como propuesta educativa de síntesis creativa para la educación superior. España: Editorial Académica Española.
}

Presente y futuro latinoamericano de la enseñanza y el aprendizaje en entornos virtuales referidos a educación universitaria. Beatriz Fainholc. 


\section{Una universidad o un programa universitario virtual para América Latina}

Es necesario definir qué es una universidad o programa universitario virtual y cuál sería su sentido en el contexto de una sociedad de conocimiento en América Latina.

La virtualidad en la universidad es hoy la propuesta de educación superior para responder lo más satisfactoriamente posible a los cambios sociales, económicos y culturales en tiempos latinoamericanos digitales.

Como se ha dicho se requieren crecientes demandas de revisión de cánones tradicionales, una formación profesionalizada, actualización y renovación permanente de los conocimientos, management flexible, etc. Internet, las TIC y sus formatos derivados, como la modalidad de investigación $2.0^{12}$, son los más interesantes para articular los programas, curricula, cursos virtuales, etc. con estos propósitos, con especial énfasis universitario, incluyendo la propuesta informal de los MOOC.

Asimismo la tendencia de construir "Digital Humanities" -Humanidades Digitales-, que si bien se halla en investigación hacia ser (o no) un campo unificado de ideas, focaliza el conjunto de prácticas virtuales convergentes. Explora y estudia las nuevas configuraciones, transmuda dónde se produce, distribuye, disemina y usa el conocimiento, a través de conceptos, herramientas, técnicas, formatos y derivados de las TIC. Las mismas alteraron aquellos procesos relativos a un conocer, ahora en un marco de variables interdependientes con las Artes, las Humanidades, la Tecnología y las Ciencias Sociales.

Las Humanidades Digitales tendrán un rol inaugural en un mundo en el cual los científicos, tecnólogos, diseñadores de software, productores de conocimiento y cultura, no podrán trabajar más aislados. Como se sabe esta necesidad de trabajo se dice, escribe y repite, pero poco aparece en la realidad. Su ámbito central son las universidades y centros de investigación, que junto a la realidad cotidiana informal, especialmente de los jóvenes, modelarán los nuevos modelos digitales de discursos no solo académicos sino organizacionales, tanto como los populares, lo que impactará en todas las esferas públicas, entre ellas, la educativa en los tiempos presentes.

Sin embargo si no se consideran los prerrequisitos y nortes enumerados que configuran un sentido oportuno para que los programas superiores virtuales sean ventajosos, seguirán además de estar alejados de los tiempos histórico-culturales, y para el caso especial de América Latina, continuar la fragmentación y hasta la marginación social.

Además, mientras la deserción en la escuela media ${ }^{13}$ sigue mostrando las cifras conocidas y publicadas ${ }^{14}$, como también los escasos estudiantes que se gradúan, la gran desigualdad y el bajo nivel de conocimientos de los estudiantes, es decir, concordante con una falta de vinculación con la vida diaria y con los empleos, -aunque los

\footnotetext{
${ }^{12}$ UCLA. (2009). "Humanidades digitales: primera, segunda y tercera ola" Digital Humanities: First, Second and Third Waves - The Digital Humanities Manifesto 2.0

http://manifesto.humanities.ucla.edu/2009/05/29/the-digital-humanities-manifesto-20/Berry,

${ }^{13}$ Siete millones de personas no terminaron la escuela secundaria, con una deserción anual de más de la mitad de los estudiantes, a pesar de su obligatoriedad en la Argentina, dada por la ley 26.206/ 2006

${ }^{14}$ Fundación Cimientos. http://cimientos.org/
}

Presente y futuro latinoamericano de la enseñanza y el aprendizaje en entornos virtuales referidos a educación universitaria. Beatriz Fainholc. 
estudiantes usan (y abusan) del aporte de Internet, de las TIC, de los teléfonos inteligentes móviles-, se demuestra que no hay claridad de un camino a reconstruir.

Hay poca conciencia de este problema, y así se generaliza, a pesar de muchos esfuerzos loables, a que el rol de la educación virtual (incluyendo la universitaria) permanezca a nivel de sus potencialidades pero con escasas concreciones de calidad y aporte socioeconómico

No se visualiza ni rescata aun, el sentido de una educación virtual.

Redefinir la riqueza y las facetas útiles existentes en las organizaciones sociales, en las instituciones educativas, y sobre todo, en los entornos virtuales creadores, -con sus flujos informacionales de Internet, las redes sociales, etc.- de una nueva plataforma formativa de aprendizaje ubicuo, no puede desaprovecharse. Otorgarle otro sentido, sería un puente socio político tecnológico entre la tecnología y la educación, y entre las necesidades personales y colectivas de la sociedad de la información con las demandas generales y específicas de satisfacción y crecimiento de la población.

No sólo la enseñanza sino sobre todo la investigación, la experimentación y el estudio riguroso y no sólo instrumental como también la apropiación tecnológica, deberían ser de relevancia para una gestión tecnológica institucional universitaria, dedicadas a profundizar el campo significativo de la virtualidad. Otorgar un espacio de relevancia, que implica la concepción ideológica de la organización, para decidir invertir en un desarrollo tecnológico educativo local, -que se vincule a la práctica global de la innovación e investigación y desarrollo-, ayudaría a la toma de decisiones sobre muchas cosas, por ejemplo.

Los programas educativos virtuales así pueden ser en sí mismos, ofertas de formación (grado y posgrado), y de proyectos de investigación y desarrollo, a través de los mismos, ser interface o bisagra del procesamiento informacional, analítica de datos telemáticos, etc., decididos por la universidad, según su perfil organizacional, la comunidad cercana y de proyección regional, internacional, etc.

Ello implica para la gestión tecnológica universitaria ${ }^{15}$, pensar en los criterios de relevancia social y de pertinencia cultural, más allá de los de eficacia instrumental y eficiencia económica de costo-beneficio,- engarzarse en especial con las TIC y redes virtuales.

El rol otorgado y practicado en la educación en general y virtual latinoamericana en general si bien existe, aunque no homogéneamente, trata de mostrar una integración de nuevos enfoques pedagógicos apoyados y basados en tecnologías, que lentamente ha comenzado a interesar a las universidades.

La agenda actual supone considerar a la educación como variable interviniente, que desmitifica a la doxa dada por el hiper-consumo tecnológico contemporáneo y otros, íntimamente relacionada con la producción de ciencia y tecnología, con conceptos y

\footnotetext{
${ }^{15}$ En este contexto, poseen relevancia mayor de desarrollo de una cultura de evaluativa en general y en especial, de la información, insumo de un diseño tecnológico, un co-diseño, etc.
}

Presente y futuro latinoamericano de la enseñanza y el aprendizaje en entornos virtuales referidos a educación universitaria. Beatriz Fainholc. 
acciones estratégicas para y de uso creador y prudente, que conduzca a un mejoramiento sostenido y productivo de la calidad de vida, y del medio ambiente ${ }^{16}$ y demás.

\section{¿A dónde es necesario ir? Qué educación superior virtual necesita América Latina}

La necesidad de América Latina requiere de concepciones nuevas (que las hay) aunque no definitivas, sobre el aprendizaje y las nuevas habilidades y competencias a desarrollar y demostrar, formar cuadros especializados profesionales, una ciudadanía lúcida y proactiva, y comprometida. Ello hace pensar necesaria (si no urgente en muchos casos) una reestructuración seria de las universidades, facultades, departamentos académicos, unidades administrativas y espacios asociados en general y en especial, a programas virtuales.

Entre los variados factores relativos pero explicativos de la evolución y estancamiento en diferentes aspectos de la región aparecen: el crecimiento del capital y la respectiva tasa de inversión, y el bajo crecimiento de la productividad. También se puede enumerar la baja incorporación reflexiva de tecnología y revisita de los enfoques organizacionales. Se piensa que lo nombrado se relaciona íntimamente con el conocimiento científico- tecnológico, y por ende, está atravesado por los procesos y resultados del sistema educativo.

Uno de los mayores desafíos que se le presenta a la educación superior en América Latina apunta a decidir qué concepción tecnológico-educativa sostendrá con coherencia los diseños, los estilos gerenciales productivos, la generación de conocimiento, cultura, etc., que son demandados por la sociedad de la información y la economía del conocimiento en la cultura digital.

Asimismo, entre otros factores que hacen al grado de integración a la economía mundial están la distribución del ingreso, el tamaño y la racionalidad ${ }^{17}$ justificada del gasto público, que afecte positivamente o no, no sólo en el funcionamiento mejorado del mercado laboral, sino en el nivel de competitividad de la región.

\section{Papel de la gestión del conocimiento virtual}

Se define la gestión del conocimiento ${ }^{18}$, como el desarrollo de un acervo de conceptos, experiencias, saberes, procedimientos, técnicas, valores, información, percepciones, etc.

\footnotetext{
${ }^{16}$ Sostenibilidad significa reorientar las políticas públicas socioeconómico- culturales, de ciencia, tecnología y educación para proteger y desarrollar integralmente a personas, grupos y organizacionales sociales.

${ }^{17}$ La racionalidad simbólico-cultural sustantiva, reemplazante de la técnico-instrumental como lógica interna de una sociedad eficientista, de estructura hegemónica de poder, que sustenta y produce el conocimiento, debe revertirse para superar su sentido consumista material y pseudo igualitario.

${ }^{18}$ FUNDIBEQ (2012) Modelo Iberoamericano de Excelencia en la Gestión V.2012. Sede en España, Fernando El Santo, Madrid (www.fundibeq.org) http://www.fundibeq.org/opencms/export/sites/default/PWF/downloads/gallery/methodology/Modelo_Ibe roamericano_General_V_2012.pdf
}

Presente y futuro latinoamericano de la enseñanza y el aprendizaje en entornos virtuales referidos a educación universitaria. Beatriz Fainholc. 
Son creados por la estructura mental del sujeto, grupos y organizaciones para interpretar, evaluar, (re)crear, imaginar y sintetizar nuevas ideas y experiencias.

Su conceptualización apunta a los activos intangibles ${ }^{19}$ que el aprendizaje individual, colectivo y organizacional, son (o deberían ser) altamente reconocidos por los tomadores de decisiones al otorgarles oportuno sentido y valor, según opción de objetivos e intereses. El desarrollo de competencias generales, específicas y transversales digitales es central en la gestión de conocimiento, aplicables a todo sector: se trata de enfrentar el riesgo de sucumbir o desaparecer.

La gestión del conocimiento, asimismo, se relaciona con una planificación flexible y verificable en la práctica de los procesos y actividades potenciadoras de una producción 2.0 en redes, de las comunidades virtuales ${ }^{20}$. Se puede afirmar que de este modo el programa educativo electrónico, -puro o combinado- de nivel superior, llegará al desarrollo hacia el aprendizaje pleno $^{21}$, con una enseñanza tecnológica para la comprensión.

Es decir, existe más y más tecnología de avanzada, cada vez más veloz, móvil e inteligente. Pero en general la gente, los profesores/as, no se hallan formados, y sus mentalidades no agregan valor a las actividades, que así, tampoco podrían sostenerse digitalmente.

La propuesta de un enfoque relacional que establece el giro epistemológico y que impacta en un giro tecnológico de las TIC y derivados muestra que el valor del conocimiento brota del espacio reticular virtual, al interior de la institución/organización, y hacia el contexto exterior dado por consorcios, alianzas, proveedores, etc. universitarios. Este concepto de gestión del conocimiento virtual implica un cambio cultural, a nivel macro, y micro social.

\section{La gestión del aprendizaje y la enseñanza a distancia virtual}

La flexibilidad en el uso de las TIC y redes para educación superior atiende a la estructuración de contenidos, en tiempos y espacios de comunicación sincrónica y asincrónica, evaluación de pares y otros, lo que disipa un encuadre jerárquico de poder, entre quienes deciden y lideran y los que son liderados, entre los que enseñan y los que aprenden, entre el diseño, la gestión y la evaluación como momentos disociados, entre el costo y el beneficio, que sólo es un análisis económico y no considera el impacto social.

Son preocupaciones constantes, aún sin muchas soluciones agraciadas y no del todo definitivas. En continua investigación en la acción, no brindan todavía una conformidad según lo esperado en procesos y resultados -con una enseñanza que muchas veces sigue siendo rutinaria y tradicional- pero ahora con tecnologías de reducido y no óptimo uso.

\footnotetext{
${ }^{19}$ Ello devendrá el "capital Intelectual” de la organización como la acumulación del conocimiento que crea valor en y para un programa educativo renovador.

${ }^{20}$ En sus diversos tipos de comunidades de aprendizaje, de práctica, de investigación, etc.), favorecidas por la distribución electrónica global de saberes e informaciones de todo tipo.

${ }^{21}$ Perkins, D. (2010). El aprendizaje pleno. Barcelona: Paidós.
}

Presente y futuro latinoamericano de la enseñanza y el aprendizaje en entornos virtuales referidos a educación universitaria. Beatriz Fainholc. 
Más aun, según los estudios realizados, no ha mejorado directamente el rendimiento ${ }^{22}$ y la calidad de los aprendizajes, o dependiendo de áreas restringidas, dentro de algunas disciplinas especificas (como en matemática/geometría, etc.).

Hoy el enfoque de aprendizaje y enseñanza virtual de mayor vigencia, es el modelo de aprendizaje centrado en el estudiante con la aplicación de la metodología de aprendizaje basado en problemas ABP, actividades didácticas generativas con experiencias de aprendizaje para la autonomía y solidaridad. Se enseña con metodologías mediadas por tecnologías para la solución de problemas, simulación de escenarios, formulación de proyectos de intervención, estudios de casos, etc. Este enfoque y metodología -ahora virtuales-, serán de proyecto de una integración curricular y transversal de las TIC y derivados, de modo que oriente a los contenidos (actualizados y relevantes profesional y socialmente), con una formación metodológica ${ }^{23}$ integral de nivel superior.

Los aprendizajes virtuales abiertos, se relacionan hoy con diseños de programas/cursos electrónicos apoyados en los "recursos educativos abiertos" (REA), o con los cursos en línea abiertos/libres $\left(\mathrm{MOOC}^{24}\right)$, como proyectos educativos en el contexto de la virtualidad en grandes, medianas y pequeñas universidades de todo el mundo, aun muy discutidos y discutibles sin valoración definitiva ${ }^{25}$.

La estructura del poder sociopolítico tradicional aún vigente se trasvasa en saberes, valores y actitudes, proceso y producto de las prácticas de enseñanza y aprendizaje, que tienden (o no) a recrearlo, a hacerlo más reflexivo ${ }^{26}$ y productivo, compartido, inclusivo socioculturalmente y sostenible a lo largo del tiempo.

Si saber es poder, el poseer conocimiento posibilita ejercerlo y auto-regularlo, autocontrol en intervenciones reflexivas respecto a la producción de conocimiento científico-tecnológico útil, en articulación a una propuesta de cambio sociocultural local y mundial, progresivo y profundo, apoyado en estructuras económico-políticas democráticas de mentalidad ejecutora. Pero no siempre es así.

Lo enunciado es válido (y con mayor énfasis) para la formación de los académicos, investigadores, y líderes de los proyectos y programas, para que su reflexión delen la acción sirva para reformulaciones no sólo de y para la práctica pedagógica mediada por tecnologías, sino para el nuevo management organizacional.

\footnotetext{
22 Estudios longitudinales desde hace varias décadas llevadas por ej. por Rusell, W. coinciden con esta apreciación: Ver: www.nondifferencesignitifacative.org

${ }^{23}$ Recursos tecnológicos de foros, Wiki, blogs, Webquest, e-porfolios, interacción en Facebook - Google groups, Skype, teléfonos inteligentes, etc, se utilizarán para la interacción y comunicación entre los estudiantes y profesores- tutores/as.

${ }^{24}$ Los cursos llegan a mas de 350 mil estudiantes o más, inscriptos por curso, mediado por una selección y combinación de recursos, basados en tecnologías móviles e inteligentes, en redes, etc que aun no se sabe su rendimiento, impacto, y duración de esta oferta universitaria, relacionada con sus costos.

${ }^{25} \mathrm{http}: / /$ webquestorgar.blogspot.com.ar/2014/02/acerca-de-los-mooc-conceptos-opiniones.html.

${ }^{26}$ La reflexividad sistémica ${ }^{26}$ es una propuesta teórica de práctica intelectual, integrante y condición para una teoría crítico-social hoy, de la sociedad red. Se caracteriza no tanto por el análisis individual sino por el sustrato colectivo socio-cognitivo, expresado a través de herramientas y operaciones analíticas, hoy disponibles en Internet y derivados. Por las vías críticas, se robustece la seguridad epistemológica del saber sociológico y se tiende a fortalecer un conocimiento científico- tecnológico, pertinente y positivo, intrínsecamente relacionado a la esfera educativa.
}

Presente y futuro latinoamericano de la enseñanza y el aprendizaje en entornos virtuales referidos a educación universitaria. Beatriz Fainholc. 
Así la formación del profesor es un capitulo muy relevante y de preocupación constante dentro del contexto innovador de una educación virtual. El replanteo de su rol (que en muchas universidades/cursos, es tutor también) se relaciona íntimamente con la concepción y acción de la "Gestión del aprendizaje".

Se han escrito ríos de tinta acerca de que el profesor debe formarse necesariamente para asumir un nuevo papel y aprender a "moverse/desempeñarse" en un entorno virtual ${ }^{27}$, trabajando en aulas virtuales, no en solitario sino en una comunidad virtual, de aprendizaje y de práctica $^{28}$, junto a los estudiantes y colegas. No abundaremos al respecto aquí.

Recordar. Los proyectos de innovaciones educativas en todos sus formatos tecnológicos, no funcionan ni terminan mal, sino que no se deciden de modo estricto, multidisciplinario, estratégico- racional y crítico, no se financian de modo sostenido, ni se piensan bien en sus sentidos, ni se planifican ajustadamente a las realidades, ni se evalúa lo que se gestiona.

Esto es lo ocurre en la mayoría de los países emergentes. Pero ello no significa resignación ni bajar los brazos, sino doblar los esfuerzos y seguir adelante ya que hoy todo proceso y relación humana pasa por la tecnología, aunque ésta sea sólo- aunque central hoy-, una parte de la Historia.

\section{Acciones educativas, científicas y tecnológicas innovadoras concretas.}

Superar la e-exclusión remite a diseñar, implementar y evaluar acciones educativas de amplia cobertura social, que en la experiencia de la autora en el trabajo con las TIC, articulando el saber científico tecnológico, inscriptas en dimensiones socio culturales, económicas y políticas de los proyectos, demuestran un aprovechamiento / apropiación de las comunicaciones electrónicas, sin límite en espacios y tiempos, ni niveles socio económicos hacia la inclusión social. La articulación con las políticas públicas científico-tecnológicas educativas conduciría a ser resolutivo en una pertinente producción de conocimiento.

Entre las acciones concretas:

- Enseñar a leer críticamente ${ }^{29}$ y a escribir estratégicamente los mensajes en cualquier formato de las pantallas de computadoras, celulares, tabletas, y otros. Ello significa:

\footnotetext{
${ }^{27}$ Hoy en boga casi con exclusividad en América Latina del software Moodle de código abierto, replantea cómo se enseña, cómo se aprende, y tender a aprender a aprender.

${ }^{28}$ Wenger, E. \& Snyder, W. (2000). Communities of practice: the organizational frontier. Harvard Business Review 139-145.

http://itu.dk/people/petermeldgaard/B12/lektion\%207/Communities\%20of\%20Practice_The\%20Organiza tional\%20Frontier.pdf - Consultado el 15 de octubre/2015

${ }^{29}$ Fainholc, B. (2006) La lectura crítica en Internet (2006) Lectura y Vida, Revista Latinoamericana de Lectura. http://www.lecturayvida.fahce.unlp.edu.ar/numeros/a26n2/26_02_Fainholc.pdf Fainholc, B (2004) Lectura crítica en Internet. Análisis y utilización de los recursos tecnológicos en educación .Editorial Homo Sapiens, Buenos Aires, Argentina. http://www.terras.edu.ar/aula/tecnicatura/3/biblio/FAINHOLC-Beatriz-La-necesid.pdf
}

Presente y futuro latinoamericano de la enseñanza y el aprendizaje en entornos virtuales referidos a educación universitaria. Beatriz Fainholc. 
ejercitar en la producción de segundas y terceras lecturas deconstructivas y de ensayos escriturales con el código digital de la web.

- Apuntar a enseñar programación (en escuelas y universidades), que significa desarrollar autonomía y protagonismo con experticia, y superar/reformular los limites y rigideces tecnológicas del software, al liberar creatividad y pertinencia con prudencia. Se trata de desmontar los mitos y falsedades informacionales existentes acerca de que los diseños se hallan en manos de especialistas.

- Crear un libro wiki masivo on-line de y para "Consultas de la ciudadanía", por temas, áreas geográficas (a pesar de la cobertura total de Internet, etc., para recurrir a las comunidades, recoger, escuchar distintas propuestas. Ello producirá una "alquimia socio -educativa y política de las multitudes" ${ }^{30}$, donde los ciudadanos/as proponen ideas, manifiestan políticas a través de Internet, que permiten acceder acerca de lo que ocurre, emitir opiniones por escrito, y votar por esas propuestas.

- Realizar "tormentas de ideas" para el ABP (aprendizaje basado en problemas), en grupos cerrados en Facebook, que permitan subir información, imágenes, etc. de todo tipo para discutir/chatear lo presentado por líderes representativos y que moldean el diseño futuro de las políticas. Publicar los resultados, y decidir con saberes profesionales estratégicos, las prioridades de acción. La época online puede hacer más transparente al gobierno, con su posibilidad horizontal si se decide por una gobernabilidad.

- Aplicar técnicas psico-socio-pedagógicas de Estudio de casos, como socio-cognitivas de inducción, toma de conciencia y resolución/provisión/anticipación de problemas, para explicarse mejor los fenómenos, y conseguir la integración/inclusión social, no solo con una asignación sostenida de recursos sino con significativa creatividad, audacia y anticipación.

- Acceder y escribir blogs, correos electrónicos y mensajes de texto acerca de múltiples informaciones, desde los antecedentes penales de los candidatos parlamentarios, y otros (en la India ya se hace), o usar los teléfonos celulares que permiten conocer, intercambiar y controlar mejor las decisiones, las elecciones (en África ya se logra), o difundir conocimiento científico- tecnológico, académico y profesional, etc., a un público global.

- Registrar en la nube virtual todas las resoluciones de las instancias de decisión para que de modo abierto a todos se pueda acceder a toda información, usarla en educación formal, etc. vía mensaje de texto, interacción directa entre usuarios o grupos de usuarios, para que políticos y ciudadanos por igual, basen sus decisiones, no más en intuiciones, sino en saberes y proyectos científico-tecnológicos, verificar si se ejecutan

\footnotetext{
(2004). Investigación: La lectura crítica en Internet: desarrollo de habilidades y metodología para su práctica. RIED. Revista Iberoamericana de Educación a Distancia, volumen 7, nº 1 y 2. [en línea] Disponible en: http://www.utpl.edu.ec/ried/images/pdfs/vol7-1-2/investigacion.pdf

(2001)La práctica de la lectura crítica en internet paso previo a la evaluación y aplicación de sus recursos. http://www.slideshare.net/abocc/lectura-en-internet

${ }^{30}$ Pisani, F. Piotet, D. (2009) La Alquimia de las multitudes. como la web está cambiando en mundo. Ediciones Paidós Ibérica. Barcelona, España.
}

Presente y futuro latinoamericano de la enseñanza y el aprendizaje en entornos virtuales referidos a educación universitaria. Beatriz Fainholc. 
o no, salvar la incoherencia entre el saber (académico-profesional) y la ejecución evaluada y contrastada en las diversas realidades, etc.

- Conocer y reconocer las manifestaciones de nuevos activismos políticos, apropiando la posibilidad de la Internet 2.0 o social y participativa, y la 3.0, donde se pueden utilizar y cruzar conceptos y estrategias para consultar y discutir la voluntad del público.

\section{Recomendaciones}

Seamos serios y rigurosos, decididos y racionales en la introducción y gestión de innovaciones de tecnología educativa en la educación universitaria con programas electrónicos puros o mixtos, sin dejar de lado que existen otras serias prioridades educativas en la región, que se favorecerían con las TIC y formatos asociados, integrados adecuadamente.

Todo es importante en materia educativa para los países emergentes que adolecen de innumerables necesidades insatisfechas, aplicadas a educación, salud, vivienda, etc. Sin embargo, la decisión de invertir a corto, mediano y largo plazo en educación (primaria y media obligatoria, como también en proyectos educativo- tecnológicos ${ }^{31}$ de capacitación y de extensión universitaria para la comunidad), redundaría en la formación de una ciudadanía local y mundial, y tendería a concretar un sistema especifico de aseguramiento $^{32}$ de la calidad para la educación superior virtual.

Entre los capítulos a considerar, pecando de ser algo reiterativos, que requieren decisiones para liderar, gestionar y evaluar los programas virtuales de educación superior, se hallan:

- Diseñar y desarrollar procesos de evaluación diagnóstica institucional (auto, co y hetero evaluación, para la introducción de innovaciones tecnológicas, previendo posibles inconvenientes y obstáculos, que pudieran surgir en las variados momentos de implementación de las decisiones;

- Robustecer las tendencias (hoy demandas sociales) de apertura, management horizontal y democrático, inversión racional y sostenida en infraestructura, investigaciones científico-tecnológicas, sistemas de evaluación y de aseguramiento de la calidad según componentes de programas con análisis del impacto social del programa virtual, como promover procesos de aprendizajes autónomos y resolutivos, activados por una enseñanza profesionalizada $\mathrm{y}$ efectiva, hoy mediada por tecnologías electrónicas, así sean de complemento a la educación cara a cara.

- Seleccionar y combinar, con interpretaciones aterrizadas socioeconómica y culturalmente, las tecnologías electrónicas soporte con relevancia cultural,

\footnotetext{
${ }^{31}$ Considerar el Informe Horizonte, UOC (.2015), en cuanto a las alusiones de la tecnología arribadas y la por venir para todos los niveles del sistema educativo.

${ }^{32}$ Fainholc, B. (2011) Capítulo: "La evaluación de los programas educativos virtuales de nivel universitario", en el Libro "El Aseguramiento de la Calidad de la Educación virtual”. Autores Rama, C y Granda, D, J .Editorial Chimbote, Perú.
}

Presente y futuro latinoamericano de la enseñanza y el aprendizaje en entornos virtuales referidos a educación universitaria. Beatriz Fainholc. 
pertinencia social, eficiencia económica, eficacia instrumental, funcionalidad, y prudencia para los programas universitarios virtuales.

- Analizar, jerarquizar, formar, redistribuir, prever los recursos humanos, tecnológicos y económico-financieros, disponibles, necesarios y a conseguir en una línea de tiempo estratégica, como una propuesta que guíe las acciones innovadoras de las TIC y las redes en los programas formativos

- Promover la investigación de las innovaciones tecnológico-educativas virtuales, favoreciendo la reflexión en la acción y para la acción continua, durante la implementación, para su ajuste y mejoramiento, dentro de una revisita de los contextos socio-organizacionales superiores, en donde se inscribirán.

- Sostener una capacitación profesional, preservar y desplegar la participación de todos los estamentos de un programa educativo superior para resolver y anticipar problemas, con resoluciones ajustables y de utilidad social, a fin de impulsar la satisfacción de necesidades y demandas, para provocar y extender el desarrollo local, regional, nacional y global, a través de programas de aprendizaje electrónico puro (o e-learning) o mixtos (o blended learning).

\section{Conclusiones abiertas}

Se concluye provisoriamente que:

- La racionalidad hermenéutica (interpretativa), de la "teoría de la acción comunicativa ${ }^{33}$ - en contraposición a la técnico-instrumental de la eficacia y la eficiencia- debería apoyar redes donde se reconstruyen y resignifican los conceptos, los sentidos y las experiencias, en contraste con la noción tecnocrática de objetividad. Los intercambios apoyan patrones de interacción comunicativa y simbólica, que conforman otros significados/contenidos de apariencia explícita e implícita.

- Recurrir a la práctica de la teoría crítica ${ }^{34}$ que analiza y explica lo social en los mitos $^{35}$ contradicciones $^{36}$ y paradojas $^{37}$ de la modernidad -ahora la híper-

\footnotetext{
${ }^{33}$ Habermas, J. (1989) Teoría de la acción comunicativa: II tomos. Madrid: Trotta.

${ }^{34}$ El concepto de Teoría Crítica incluye no sólo las exigencias metodológicas para estudiar un determinado objeto sino que persigue un carácter emancipador en su orientación. Los "por qués" y los "para qués" son atribuciones determinantes para orientar sus fines. La búsqueda de verdades (ya que todas son aceptadas en honor a su relatividad contextual) está orientada por valores. La aproximación al conocer, no se reduce al acercamiento lineal de un sujeto pensante a un objeto de investigación, sino que se parte del principio que los procesos de pensamiento, del conocer y actuar, están ya condicionados por los mismos objetos. Los conocimientos son dialécticos e históricos, y no prevalecen unos sobre otros, sino por una explicación de relación interdisciplinar. No se trata de imponer un conocimiento crítico, sino de proporcionar instrumentos de reflexión crítica, donde se respetan los aportes de todos los participantes en un clima de comunicación y de análisis comprometido.

${ }^{35}$ Mito se representa borrosamente en los límites de la inteligibilidad. Su función simbólica que los explica, coadyuva a la cohesión socio-cultural. Son formulaciones que permiten enfrentar lo incierto /informulable, con una pretensión de certeza o congruencia. Los contextos político-sociales los legitiman, dependiendo de las circunstancias.
}

Presente y futuro latinoamericano de la enseñanza y el aprendizaje en entornos virtuales referidos a educación universitaria. Beatriz Fainholc. 
modernidad- y desnudar la doxa, el estereotipo, etc., de la vida cotidiana como de los modelos digitales hegemónicos. La ciudadanía no puede / no ha sido formada en la mentalidad reticular reflexiva (y menos digital) para des-cubrir mitos, paradojas y contradicciones del sistema socio-político-económico y tecnológico existente: tarea educativa, co-protagonista para la inclusión social.

- Trabajar hacia una epistemología integrada y participativa al aprovechar las posibilidades tecnológicas de TIC y redes, con acciones colectivas para revertir (in)decisiones ideológicas no comprometidas y de pretensión (ilusión) de mejoramiento de la calidad de vida, al anticipar alternativas para un futuro. Al aumentar el acceso, la alfabetización tecnológica ayuda a la cohesión/integración sociocultural, -con respaldo del saber y operar científico-tecnológico y educativo, contrastado, para el convivir ${ }^{38}$ y decidir en un mundo global informacional y de cultura digital, que hay que enseñar para criticarla, y aprenderla para dominarla.

- Reconocer que la sociedad en su conjunto forma/educa: la responsabilidad de la trans-mediática electrónica es visibilizar los comportamientos de todos los ciudadanos, los gobernantes, legisladores, representantes organizaciones sociales, etc. Ni la educación, ni la ciencia ni la tecnología no son neutras ${ }^{39}$ ni autónomas porque se configuran de acuerdo con la dominancia histórico sociocultural.

Los responsables formativos de una sociedad frente al nuevo escenario de la conectividad digital, deberían preguntarse cuánto de lo que se viene haciendo en términos sociales, tecnológicos, sirve; y cuánto debe ser modificado, ajustado, sin aferrase a dogmatismos, para el diseño y ejecución de programas transformadores, con la demostración de las nuevas competencias aprendidas que modifican el repertorio cultural limitado ${ }^{40}$.

- La tecnología, se dijo, sólo es una parte de la historia: formación y capacitación de líderes, managers, profesores/as y los perfiles de las nuevas figuras profesionales que reclaman los entornos y programas virtuales, en relación a ejecutar un papel más pertinente para las comunidades más marginadas. Otorgar más educación y capacitación para una absorción ocupacional productiva en las nuevas

Por ejemplo: las historias se fueron construyendo narrativas sociales muchas de las cuales responden al determinismo tecno-científico supuestamente progresista de la calidad de vida, mientras otras han sido libertarias y críticas de la tecno ciencia.

${ }^{36}$ Una contradicción implica el antagonismo entre dos o más proposiciones, su afirmación y su negación, los que nunca pueden ser verdaderos al mismo tiempo. Ejemplo: más información ayuda a conocer más sobre el mundo global, pero ello no ayuda a reflexionar, a discriminar información veraz, especialmente cuando se eliminan las voces de aquéllos que no acuerdan en los pensamientos de moda vigente, o se manipula la información según razones ideológicas.

${ }^{37}$ Paradojas es lo opuesto a lo que una persona considera cierto/verdadero.

Ejemplo: A pesar de la popular y requerida navegación de muchos sitios y blogs, los usuarios, generalmente, no recuerdan los nombres, direcciones de los mismos. Tampoco les preocupa si la información es de fuentes serias y autorizadas, o en reconocer que razones impulsan su presencia (influencias políticas, tarifas bajas de artículos, búsqueda de popularidad, etc.

${ }^{38}$ Touraine, A. (2000). Crítica de la Modernidad. Bogotá: FCE.

${ }^{39}$ Feenberg. A. (2005). Teoría crítica de la tecnología. Revista Iberoamericana de ciencia, tecnología y sociedad-CTS. Vol.2,N5.pp109-123

${ }^{40}$ Bauman, Z. (2013). La cultura en el mundo de la modernidad líquida. Buenos Aires: Fondo de Cultura Económica.

Presente y futuro latinoamericano de la enseñanza y el aprendizaje en entornos virtuales referidos a educación universitaria. Beatriz Fainholc. 
especialidades con una dosificación del superficial y efímero bombardeo tecnológico consumista son medidas serias.

- La concepción de la inclusión social se vincula con distribuir de modo justo los conocimientos e interacciones disponibles en la sociedad. Entiende que las desigualdades sociales son "producidas" y que pueden ser eliminables ${ }^{41}$ a través de un esfuerzo político. Destrabar los conflictos limitativos y reformular los problemas de toma de decisiones sobre fines y objetivos, de representatividad, de gobernabilidad, de gestión y de contrastación en la realidad de los procesos, resultados e impactos alcanzados (o no) por los programas gubernamentales sociales beneficiosos y centrados en / para la ciudadanía, es una necesidad. Ello con una conciencia acerca del trabajo colaborativo comprometido, que quiebra la lógica compartimentada de la administración tradicional.

Entonces: la contribución del área de la ciencia, las tecnologías inscriptas en una sociedad y cultura en su relación con la educación digital, debe tender a una racionalidad tecnológica sustantiva, hacia una utopía social útil/productiva, con políticas públicas científico-tecnológico-educativas robustas. Una genuina, relevante toma de decisiones para formar gente resolutiva para resolver/anticipar problemas, rescatar/desmitificar la memoria colectiva articulando procesos y productos innovadores, fortalece alternativas equitativas para superar exclusiones por la adaptación reflexiva de ideas and acciones imaginativas, de inventiva y críticas.

\section{Incógnitas}

En la era digital, con sus propuestas fascinantes, escurridizas, deslizadas, también brinda la posibilidad de impresionantes y desconocidas oportunidades. Las redes sociales virtuales para la producción y distribución, con una acumulación ${ }^{42}$ y horizontal procesamiento de la información en línea, que hasta hace poco tiempo no existían. Sin embargo este potencial carga mitos y paradojas a ser desarmadas por acciones educativas electrónicas explicitas.

Pero continúan los interrogantes, que iluminan nuevas preguntas:

- ¿En qué valores se decidirán/tomarán decisiones de políticas públicas para la producción de conocimiento científico tecnológico y educativo para construir un futuro político democrático y pluralista?

- Con las TIC sociales, Facebook, un gobierno 2.0 con el uso de las redes sociales virtuales globales como oportunidades poco explotadas, ¿servirán para seleccionar, cuestionar líderes, administradores públicos y decisores políticos, colaborando en la consulta ciudadana/ vecinal, para una democracia genuinamente participativa, para el presente y en prospectiva constructora a medio y largo plazo?

\footnotetext{
${ }^{41}$ Bobbio, N. (1996). El filósofo y la politica. Antología. México: FCE.

${ }^{42}$ Fainholc, B. (2015) http://webquestorgar.blogspot.com.ar/2015/03/big-data.html http://webquestorgar.blogspot.com.ar/2014/11/internet-de-las-cosas-2-pensar-riesgos.html
}

Presente y futuro latinoamericano de la enseñanza y el aprendizaje en entornos virtuales referidos a educación universitaria. Beatriz Fainholc. 
- El uso interactivo de las redes ${ }^{43}$, defenderán políticas de apertura horizontal, o puntos de vista defensores del statu quo? La imagen democrática e inclusiva de las TIC, ¿ocultará las inequidades y la e-exclusión social (etnias, género, nivel socioeconómico, geografía, edad, etc.)?

- El impacto de las fortalezas y debilidades de la producción global del saber remarca inequidad, vulnerabilidad y riesgos que necesitan una redefinición del diseño, implementación y evaluación de las políticas públicas socio-económico- culturaleducativo y científico-tecnológico; ¿qué revisará la estructura conflictiva del poder?

Se concluye: se trata de tareas pendientes para diseñar y recrear el salto histórico de los modelos prescriptos a los fluidos, para concretar puentes decididamente democráticos.

Las conquistas en la historia se hicieron con la tecnología del arado, la espada y el libro. Hoy, con ciencia, tecnología y educación con y en la web y los teléfonos móviles inteligentes en conectividad.

Presentación del artículo: 9 de diciembre de 2015 Fecha de aprobación: 11 de diciembre de 2015 Fecha de publicación: 30 de enero de 2016

Fainholc, B. (2016). Presente y futuro latinoamericano de la enseñanza y el aprendizaje en entornos virtuales referidos a educación universitaria. RED. Revista de Educación a Distancia. Número 48(2). Consultado el (dd/mm/aaaa) en http://www.um.es/ead/red/48

\section{Bibliografía}

Bauman, Z. (2013). La cultura en el mundo de la modernidad líquida. Fondo de Cultura Económica, Buenos Aires: Argentina.

Bates, T. (2003). La gestión del cambio tecnológico. Barcelona: Gedisa.

Bobbio, N. (1996). El filósofo y la política. Antología. México: Editorial FCE.

Bourdieu, P. (1991). El sentido de la práctica. Madrid: Taurus.

Bourdieu, P. (1997). Razones prácticas. Barcelona: Anagrama.

Bourdieu, P. (1984). Espace social et genèse des "classes". Actes de la recherche en sciences sociales. Año 1984. Volumen 52. Numéro 1. pp. 3-14.

\footnotetext{
${ }^{43}$ Fainholc, B. Facebook, https://www.facebook.com/beatriz.fainholc Fainholc, B (2011) Un análisis contemporáneo de Twitter http://webquestorgar.blogspot.com.ar/2011/06/revista-de-educacion-distancia.html?view=magazinetwitter Fainholc, B. (2014) Selfies http://webquestorgar.blogspot.com.ar/2014/05/los-selfie.html?view=magazine
}

Presente y futuro latinoamericano de la enseñanza y el aprendizaje en entornos virtuales referidos a educación universitaria. Beatriz Fainholc. 
Bruner, J. (1999). La educación, puerta de la cultura. Buenos Aires: Paidós.

Buckingham, D. (2008). Repensar el aprendizaje en la era de la cultura digital. Buenos Aires: Manantial.

Buehring, S. (2013, febrero 13). Project Management Success with the Top 7 Best Practices. [Blog]. Articles.Send2.Us. Recuperado de http://articles.send2.us/projectmanagement-success-with-the-top-7-best-practices/

Burbules, N y Rupert, B. (1999) Critical Thinking and Critical Pedagogy: Relations, Differences, and Limits. En Popkewitz, T. y Fendler, L. (Ed). Critical Theories in Education, Thomas S. Nueva York: Routledge. Recuperado de http://faculty.education.illinois.edu/burbules/papers/critical.html

Burbules, N. y Callister, T. (2001). Riesgos y promesas de la sociedad de la información y el conocimiento. Buenos Aires.

Bunge, M. (2004): Mitos, hechos y rezones: cuatro estudios sociales. Buenos Aires: Sudamericana.

Castells, M. (2002, abril 10) "La dimensión cultural de Internet". [Serie de Debates]. Cultura XXI. Sesión I. Instituto de Cultura. Barcelona: UOC. Recuperado de http://www.uoc.edu/culturaxxi/cat/articles/castells0502/castells0502_imp.html

CEPAL (2011). Desafíos para una educación con equidad en América Latina y el Caribe. Encuentro Preparatorio Regional 2011. Naciones Unidas - Consejo Económico y Social. Revisión Ministerial Anual.

Berry, D. (2011, enero 14). Digital Humanities: First, Second and Third Waves. [Blog] Stunlaw. Recuperado de http://manifesto.humanities.ucla.edu/2009/05/29/thedigital-humanities-manifesto-20/Berry

ECOSOC- RMA. CINDA. (2007). Educación superior en Iberoamérica. Informe 2007. Santiago, CINDA - RIL.

Fainholc, B. (2001). La práctica de la lectura crítica en internet paso previo a la evaluación y aplicación de sus recursos. [Artículo en línea] Recuperado de http://www.slideshare.net/abocc/lectura-en-internet

Fainholc, B. (2004, septiembre 6) "El concepto de mediación en la tecnología educativa apropiada y crítica". [Blog] EDUC.AR. http://portal.educ.ar/debates/educacionytic/nuevos-alfabetismos/el-concepto-demediacion-en-la-tecnologia-educativa-apropiada-y-critica.php

Fainholc, B. (2004). Lectura crítica en Internet. Análisis y utilización de los recursos tecnológicos en educación. Buenos Aires: Homo Sapiens. Recuperado de http://www.terras.edu.ar/aula/tecnicatura/3/biblio/FAINHOLC-Beatriz-Lanecesid.pdf

Presente y futuro latinoamericano de la enseñanza y el aprendizaje en entornos virtuales referidos a educación universitaria. Beatriz Fainholc. 
Fainholc, B. (2004). Investigación: La lectura crítica en Internet: desarrollo de habilidades y metodología para su práctica. En RIED. Revista Iberoamericana de Educación a Distancia, volumen $7, \mathrm{n}^{\circ} 1$ y 2. [en línea]. Disponible en: http://www.utpl.edu.ec/ried/images/pdfs/vol7-1-2/investigacion.pdf

Fainholc, B. (2006). La lectura crítica en Internet. En Lectura y Vida, Revista Latinoamericana de Lectura. Año 26. Número 2. La Plata: Facultad de Humanidades y Ciencias de la Educación de la Universidad Nacional de la Plata. Recuperado de http://www.lecturayvida.fahce.unlp.edu.ar/numeros/a26n2/26_02_Fainholc.pdf

Fainholc, B. (2009). Programas, profesores y estudiantes virtuales. Buenos Aires: Santillana.

Fainholc, B. (2011). Distance and Flexible Education Applying ICT for an Innovative and Open Learning, New York: Nova Science.

Fainholc, B. (2011). Contradicciones y dilemas tecnológico-educativos: la búsqueda de coherencia epistemológica y calidad de los programas educativos virtuales entre su diseño y la práctica. Revista VEC Virtualidad, Educación y Ciencia, Número 1. Córdoba: Centro de Estudios Avanzados, Universidad Nacional de Córdoba. Recuperado de http://revistas.unc.edu.ar/index.php/vesc/article/viewFile/333/332

Fainholc, B. (2011). Un análisis contemporáneo del Twitter. RED, Revista de Educación a Distancia. Número 26. 15 de mayo de 2011. Recuperado de http://www.um.es/ead/red/26/

Fainholc, B. (2011). Una tecnología educativa apropiada y crítica. Nuevos enfoques. España: Editorial Académica Española.

Fainholc, B. (2011). "La evaluación de los programas educativos virtuales de nivel universitario", en Rama, C y Granda, D, J, El Aseguramiento de la Calidad de la Educación virtual. Perú: Editorial Chimbote.

Fainholc, B. (2012). Aprendizaje electrónico mixto. El blended learning como propuesta educativa de síntesis creativa para la educación superior. España: Editorial Académica Española.

Fainholc, B. (2014, mayo 5). Selfies. [Blog]. Tecnología educativa apropiada y crítica. Recuperado de http://webquestorgar.blogspot.com.ar/2014/05/losselfie.html?view=magazine

Fainholc, B. (2015, marzo 16). Big data. [Blog]. Tecnología educativa apropiada y crítica. Recuperado de http://webquestorgar.blogspot.com.ar/2015/03/big-data.html

Fainholc, B. (2014, noviembre 17). Internet de las cosas. [Blog]. Tecnología educativa apropiada y crítica. Recuperado de http://webquestorgar.blogspot.com.ar/2014/11/internet-de-las-cosas-2-pensarriesgos.html

Presente y futuro latinoamericano de la enseñanza y el aprendizaje en entornos virtuales referidos a educación universitaria. Beatriz Fainholc. 
Feenberg, A. (2009). Diez paradojas de la Tecnología. [Conferencia] Recuperado de https://www.sfu.ca/ andrewf/books/Span_Ten_Paradoxes_of_Technology.pdf

Feenberg, A. (2005) Teoría crítica de la tecnología. Revista Iberoamericana de ciencia, tecnología y sociedad, $N^{o}$. 5, págs. 109-123. Disponible en http://dialnet.unirioja.es/servlet/articulo?codigo=2358086 Acceso 27-03-2014

Ferguson, R. (2011). Towards a social learning space for open educational resources. The Open University. Recuperado de http://kmi.open.ac.uk/people/member/rebeccaferguson

Foucault, M. (2006). Territorio, Seguridad y Poder. México: FCE. .

Foucault, M. (1995). Un diálogo sobre el poder. Madrid: Alianza.

Fullan, M. (2002). Los nuevos significados del cambio en la educación. Barcelona: Octaedro.

FUNDIBEQ. (2012). Modelo Iberoamericano de Excelencia en la Gestión V.2012. Sede en España, Fernando El Santo, Madrid. Recuperado de http://www.fundibeq.org/opencms/export/sites/default/PWF/downloads/gallery/meth odology/Modelo_Iberoamericano_General_V_2012.pdf

Habermas, J. (1982). La acción comunicativa Barcelona: Taurus.

Habermas, J. (1989). Teoría de la acción comunicativa. Madrid: Trotta.

Habermas, J. (1991). Conciencia moral y acción comunicativa. Barcelona: Península.

Perkins, D. (2010). El aprendizaje pleno. Barcelona: Paidós.

Piketty, T. (2014). El capital en el siglo XXI. México: FCE.

Pisani, F. y Piotet, D. (2009). La Alquimia de las multitudes. Como la web está cambiando en mundo. Barcelona: Ediciones Paidós Ibérica.

Popkewitz T. (1994). Modelos de poder y regulación social en pedagogía. Barcelona: Pomares Corregidor.

Senge, P. (2002). Las organizaciones que aprenden. Bogotá: Editorial Norma.

Tabares Quiroz, J. y Correa Vélez, S. (2014). Tecnología y sociedad: una aproximación a los estudios sociales de la tecnología. En Revista Iberoamericana de Ciencia Tecnología y Sociedad, Vol.9, $N^{\circ}$ 26. Recuperado de http://www.revistacts.net/files/Volumen_9_Numero_26/Tabares_EDITADO.pdf

Touraine, A (2000). Crítica de la Modernidad. Bogotá: FCE.

Presente y futuro latinoamericano de la enseñanza y el aprendizaje en entornos virtuales referidos a educación universitaria. Beatriz Fainholc. 
UNESCO-IIEP (2007). Herramientas para la gestión de proyectos educativos con TIC Serie de publicaciones del Proyecto @lis-INTEGRA. Argentina. Recuperado de http://unesdoc.unesco.org/images/0015/001580/158068s.pdf

UNESCO. Situación Educativa de América Latina y el Caribe: Hacia la educación de calidad para todos al 2015. Recuperado de http://www.unesco.org/new/fileadmin/MULTIMEDIA/FIELD/Santiago/images/SITI ED-espanol.pdf

Wenger, E. y Snyder, W. (2000, enero/febrero). Communities of practice: the organizational frontier. En Harvard Business Review, p. 139-145. Recuperado de http://www.rareplanet.org/sites/rareplanet.org/files/Communities_of_Practice_The_ Organizational_Frontier\%5B1\%5D.pdf

Wolton, D. (2000). Internet, ¿y después? Barcelona: Gedisa. 